\title{
Regional Distribution of Carbon Intensity and its Driving Factors in China: An Empirical Study Based on Provincial Data
}

\author{
Rongwei Wu ${ }^{1,2}$, Jiefang Dong ${ }^{3}$, Liang Zhou ${ }^{4,5 *}$, Lu Zhang ${ }^{1,2}$ \\ ${ }^{1}$ State Key Laboratory of Desert and Oasis Ecology, Xinjiang Institute of Ecology and Geography, \\ Chinese Academy of Sciences, Urumqi 830011, China \\ ${ }^{2}$ University of Chinese Academy of Sciences, Beijing 100049, China \\ ${ }^{3}$ Department of Economics and Management, Yuncheng University, Yuncheng 044000, China \\ ${ }^{4}$ Faculty of Geomatics, Lanzhou Jiaotong University, Lanzhou 730070, China \\ ${ }^{5}$ Institute of Geographic Science and Natural Resources Research, CAS, Beijing 100101, China
}

Received: 18 May 2017

Accepted: 10 August 2017

\begin{abstract}
The regional distribution and driving factors of total carbon emissions have been the focus of considerable research. However, carbon intensity rather than total carbon emissions has been selected as the emissions reduction index in China. The Chinese government has committed to reducing carbon intensity by $60-65 \%$ from 2005 levels. Currently, limited academic attention has been given to the regional distribution and driving factors of carbon intensity. To explore the means of achieving the carbon intensity target in China, Gini coefficients were employed in this paper to investigate regional differences in carbon intensity across 30 provinces from 1995 to 2014. Moreover, the FGLS (feasible generalized least squares) method was applied to identify the key influencing factors of carbon intensity at the national and three regional levels. The results indicate that:

1. Chinese inter-provincial Gini coefficients of carbon intensity have increased steadily in recent years, which indicates that the difference in carbon intensity between provinces in China has widened.

2. Economic growth, foreign direct investment, and trade openness were negatively correlated with carbon intensity. Conversely, coal consumption, industrial proportion, and urbanization were positively correlated with carbon intensity. Moreover, urbanization has proven to be the most important factor affecting China's carbon intensity.

3. The dominant cause of carbon intensity varies by region. In particular, the dominant cause of carbon intensity in low- and medium-level regions is urbanization. However, the dominant cause of carbon intensity in high-level regions is coal consumption.
\end{abstract}

*e-mail: zhougeo@126.com 
4. Based on these empirical findings, policy recommendations to reduce carbon intensity were proposed. In summary, the improvement of urbanization quality in both low- and medium-level regions is urgently needed. However, optimizing the energy structure is essential to carbon intensity reduction in high-level regions.

Keywords: carbon intensity, Gini coefficient, panel date analysis, FGLS, China

\section{Introduction}

Carbon dioxide is a significant factor contributing to global climate change, and the reduction of carbon dioxide emissions has therefore become a common goal for the international community dedicated to addressing climate change on a global scale [1]. In 2014 China produced $28.06 \%$ of global carbon dioxide emissions [2]. As a responsible developing country, China has made a commitment to the world that its carbon dioxide emissions will peak in 2030, after which carbon dioxide emissions per unit of GDP (also referred to as carbon emission intensity) will be reduced $60-65 \%$ of 2005 values [3]. This goal has been reallocated to each province by setting up more specific targets. However, China is a vast country with marked regional differences in energy consumption among provinces [4-5]. Moreover, these regional differences pose a serious challenge to meeting the scheduled carbon emissions goal [6-7]. A better understanding of carbon emissions intensity (CI) and its driving factors among provinces will contribute to developing scientific and practical emissions reduction policies to achieve China's CI goal.

Previous literature mainly focused on two aspects of CI: regional differences and driving factors. Some researchers have argued that energy intensity (EI) can represent most information in CI. Hence, there has been sufficient research focusing on the regional differences in EI, drawing many meaningful conclusions. These studies can be broadly divided into two general types. Indexes, including degree of concentration (the Gini and Theil), are used by the first type to analyze the inequality of EI [8-14]. For instance, by utilizing the Theil index and its decomposition by group, Duro [10] studied inequality in energy intensity in 1990-2011 and found that generalized reduction in energy intensities on a global level coincided with an improvement in their international equality. Since then, Theil's second measure has been used by Alcantaraa and Duro [13] for analyzing international energy intensity differences between OECD countries. Furthermore, this study examined the contributions of different groups of countries to this inequality. A similar study was also conducted by Yue et al. [15]. The second research type focuses on the convergence on energy intensity [16-20]. One example of such scholarship is the research by Burnett and Madariaga [19], who extends a neoclassical growth model and examines the implications for convergence in economic growth and energy intensity across U.S. states. They found strong evidence of convergence in energy intensity among a set of advanced economies. Moreover, Herrerias [21] investigated the convergence process of EI on a global scale over the period 1971-2008, finding that developing countries converge at higher energy intensity ratios, while in the case of developed countries there are at least two convergence groups. Similar studies were also undertaken in China by Zhao et al. [17], who found that CI were converging in Chinese provinces. Overall, only limited academic attention has been given to regional differences in CI.

With regard to the driving factors of $\mathrm{CI}$, research is increasingly being conducted in different countries and regions. Various methods have been used to examine these factors: the LMDI method [22-24], the IDA method [25-26], the IPAT model [27], and the STIRPAT model [28] being the most popular. The decomposition method is an important tool employed in exploring the determinants of carbon intensity. Using the LMDI method, Zhang et al. [22] explored the driving factors of CI in 29 Chinese provinces and found that energy consumption intensity played an important role in the rapid decrease in CI from 1995 to 2012. Similar studies were also undertaken in Portugal by Robaina Alves and Moutinho [29]. Compared with the LMDI decomposition method, regression analysis and comparative analysis provide comprehensive results about each factor. Based on an improved STIRPAT regression model, taking China as an example, Wang et al. [28] investigated key factors affecting $\mathrm{CI}$ at the national level and at eight economic regional levels in China. They found that there were obvious differences in regional development; in different regions, the relationship between the variables is different. Therefore, each region should pay heed to its own particular characteristics.

As mentioned above, most previous literature considers energy intensity instead of carbon intensity as the research object, which weakens its utility for policy implications in China. Moreover, much literature focuses separately on the regional differences or influencing factors of CI. Limited research has combined the regional differences with the driving factors of CI. In addition, the majority of research has divided China using three economic regions, ignoring the significant disparity of CI among provinces. To overcome these shortcomings, this article first calculated the Gini coefficients of CI in 30 provinces in China during 1995-2014. Then the 30 provinces were ranked and divided into three groups: low-, medium-, and high-level regions. Finally, by using the FGLS method based on panel data, this paper further investigates the factors affecting $\mathrm{CI}$ at the national and three regional levels. 


\section{Materials and Methods}

\section{Regional Differences in Carbon Intensity}

Measuring regional differences in the $\mathrm{CI}$ of fossil energy consumption is the first step in researching carbon intensity reduction. There are several ways to examine regional differences in CI, such as the Theil index, coefficient of variation (CV), and Gini coefficient (Gini) - with each method having pros and cons. Therefore, this paper measured the dispersion and concentration of CI by combining $\mathrm{CV}$ with Gini [30]. The formulas for the $\mathrm{CV}$ and Gini coefficient are as follows:

$$
\begin{aligned}
& C V=\frac{\sqrt{\sum_{i=1}^{n}\left(y_{i}-\mu\right)^{2} / n}}{\mu} \\
& G=\frac{1}{2 n^{2} \mu} \sum_{i} \sum_{j}\left|y_{i}-y_{j}\right|
\end{aligned}
$$

...where $y_{i}$ is the $\mathrm{CI}$ in province $i, \mu$ denotes the national average $\mathrm{CI}$, and $\mathrm{n}$ represents the number of provinces.

\section{Panel Unit Root Tests}

Regression conducted in relation to non-stationary variables may produce spurious regression [31]. As such, the balanced panel data should be subjected to a unit root test to prove the stationarity of the variables. Panel unit root tests are widely used because they are more powerful than tests based on normal time series and cross-sectional data [32]. The methods for unit root testing panel data include the LLC, IPS, ADF, PP, and Hadri tests [28].

\section{Panel Co-Integration Tests}

The Pedroni and Kao tests are typically carried out to test co-integration relationships among variables for panel data [32]. These two approaches have good performance when testing time sequences over a short period. The null hypothesis of the Pedroni and Kao tests is that there is no co-integrational relationship between variables.

\section{Model of Driving Factors}

The IPAT (impact $=$ population $\times$ affluence $\times$ technology) identity was first proposed in the early 1970s. It is widely utilized and has been regarded as an easily understandable framework for analyzing the influence of human factors on the environment. It can be described with the following equation:

$$
\mathrm{I}=\mathrm{PAT}
$$

...where I represents the environmental pressure indicator (i.e., $\mathrm{CI}$ in this study), $\mathrm{P}$ is the total population,
A indicates the average affluence, and $\mathrm{T}$ refers to the level of technological development.

However, the obvious flaw of the IPAT model is that it can only analyze a limited number of variables. To overcome its disadvantages, York et al. [33] reformed the IPAT model into a random form and established the STIRPAT (stochastic impacts by regression on population, affluence and technology), which is described as:

$$
\mathrm{I}=\mathrm{aP} \mathrm{P}^{\mathrm{b}} \mathrm{A}^{\mathrm{c}} \mathrm{T}^{\mathrm{d}} \mathrm{e}
$$

...where a is a constant term; I, P, A, and T have the same meanings as those in Eq. (3); b, c, and d are the exponential terms of $\mathrm{P}, \mathrm{A}$, and $\mathrm{T}$, respectively; and $\mathrm{e}$ is the error term of the model.

The STIRPAT model is a multivariable nonlinear model and is often used in double logarithmic form:

$$
\mathrm{InI}=\mathrm{a}+\mathrm{bln}(\mathrm{P})+\operatorname{cln}(\mathrm{A})+\mathrm{d} \ln (\mathrm{T})+\mathrm{e}
$$

Because the STIRPAT model can be expanded to include more influencing factors, this method became a well-known technique that was widely used to explore the influencing factors of the environment. Depending on different research needs and targets, the STIRPAT model allows for the introduction of additional factors to carry out different kinds of empirical research. Considering the characteristics of energy consumption in China and learning from relevant research, we expanded the STIRPAT model by incorporating GDP per capita [34], proportion of industries [11], coal consumption [3], urbanization level [3], foreign direct investment [28], and trade openness [11] into the model. Then the STIRPAT model in this study was described as follows:

$$
\begin{gathered}
\mathrm{InCI}=\mathrm{a}+\mathrm{b} \ln (\mathrm{EI})+\mathrm{cln}(\mathrm{SI})+\mathrm{d} \ln (\mathrm{CP})+ \\
\quad \mathrm{fln}(\mathrm{URL})+\mathrm{g} \ln (\mathrm{TO})+\mathrm{h} \ln (\mathrm{FDI})+\mathrm{e}
\end{gathered}
$$

...where CI is the carbon intensity that indicates the impact on the environment, EL refers to the GDP per capita and is introduced to analyze the influence of economic growth on environmental pressure, SI is the share of industrial value-add in the GDP and is introduced to study the influence of industrial structure change on $\mathrm{CI}, \mathrm{CP}$ is the proportion of coal consumption and is used to study the influence of energy structure change on CI (because coal has the highest carbon emissions coefficient among all primary energy sources), and UL represents urbanization level and is used to study the influence of urbanization on CI. Two additional factors, namely TO (total import and export volume in overall GDP) and FDI (foreign direct investment in overall GDP) are used to study the influence of trade liberalization on CI. In Eq. (6), a is a constant; b, c, d, f, g, and h are coefficients of each variable; and e is the random error term. 
Table 1. Statistical description of variables in China, 1995-2014.

\begin{tabular}{|c|c|c|c|c|c|c|}
\hline Symbol & Variable & Unit & Mean & Std. Dev & Min & Max \\
\hline CI & Carbon emission intensity & $\mathrm{t} / 10,000$ Yuan & 0.41 & 0.31 & 0.08 & 2.44 \\
\hline EL & Economic level & Yuan/person & $18,632.44$ & $15,550.10$ & $2,316.35$ & $88,853.60$ \\
\hline IP & Industry proportion & $\%$ & 45.19 & 0.08 & 19.74 & 59.05 \\
\hline CP & Coal proportion & $\%$ & 62.85 & 0.15 & 14.26 & 90.44 \\
\hline URL & Urbanization level & $\%$ & 45.73 & 16.07 & 20.39 & 89.61 \\
\hline TO & Trade openness & $\%$ & 30.93 & 0.39 & 0.34 & 205.12 \\
\hline FDI & Foreign direct investment & $\%$ & 3.10 & 3.12 & 0.05 & 24.25 \\
\hline
\end{tabular}

\section{Data Description}

In this paper, the study period ranges from 1995 to 2014. CI is total carbon emissions divided by GDP. The data on carbon emissions used in this research was derived from the International Energy Agency (IEA) [2]. Because $\mathrm{CO}_{2}$ emissions data for each province in China are released neither by the IEA nor the Chinese Bureau, we calculated the $\mathrm{CO}_{2}$ emissions in each province based on the method proposed by Yue [15]. Moreover, we selected GDP per capita to indicate economic level. The urbanization level was calculated by dividing the urban population by total population. Data related to the industry proportion were calculated by dividing industry-added value by overall GDP. Meanwhile, the coal proportion is the ratio of coal consumption to total primary energy consumption. Foreign direct investment and trade extroversion are the proportion of actual use of foreign direct investment and total import and export volume in GDP, respectively. All the data for GDP, total population, industry-added value, and total import and export volume were obtained from the China Statistical Yearbook (1996-2015) [35] and the data on foreign direct investment came from the China Statistical Yearbook of Commerce (2015) [36]. The panel data used in this research was selected from 30 provincial statistical yearbooks in China (except for Tibet, Hong Kong, and Macao; note that Chongqing was established in 1997, so the data for Chongqing in 1995 and 1996 were back-calculated using its average growth rate [28]). To eliminate the effects of inflation on GDP, this research used GDP at 1995 levels. The statistical descriptions of

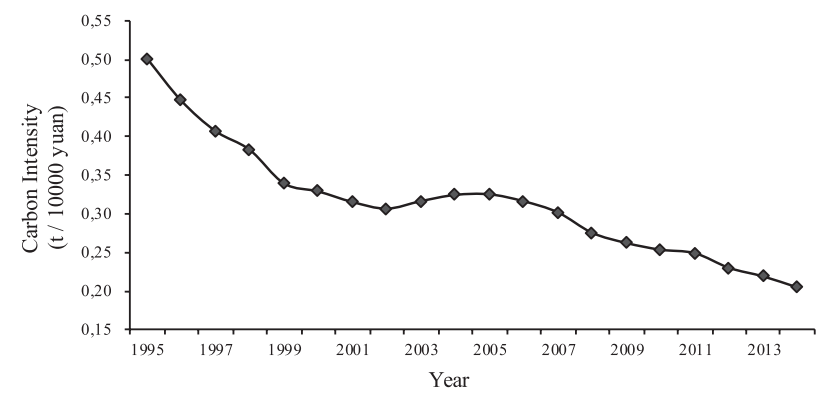

Fig. 1. CI of China 1995-2014. all variables in this research are shown in Table 1. Means of CI, EL, IP, CP, URL, TO, FDI are $0.41 \mathrm{t} / 10,000$ yuan, $18,632.44$ yuan/person, $45.19 \%, 62.85 \%, 45.73 \%, 30.93 \%$, and $3.10 \%$, respectively, among which CI ranges from $0.08 \mathrm{t} / 10,000$ yuan to $2.44 \mathrm{t} / 10,000$ yuan. EL ranges from $2,316.35$ yuan/person to $88,853.60$ yuan/person. FDI has
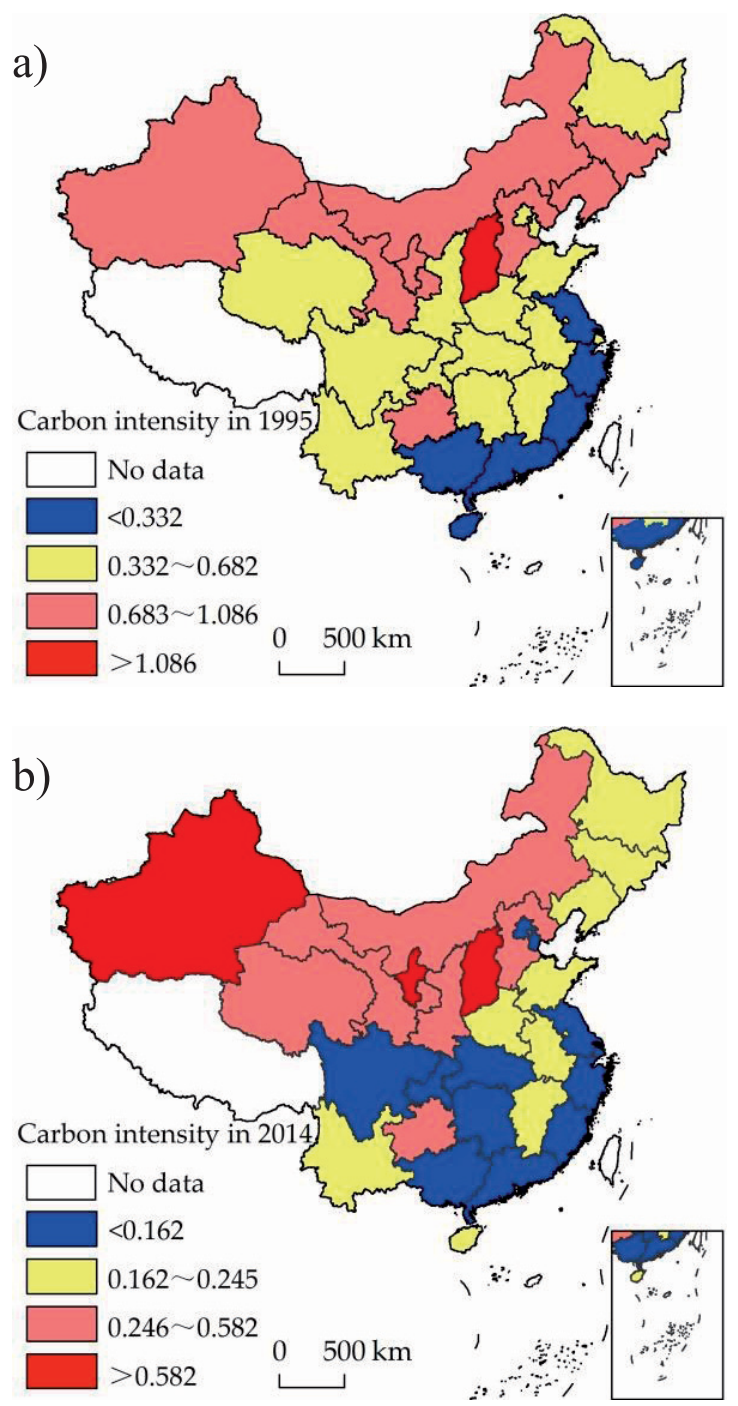

Fig. 2. Regional distributions of carbon intensity in: a) 1995 and b) 2014 . 


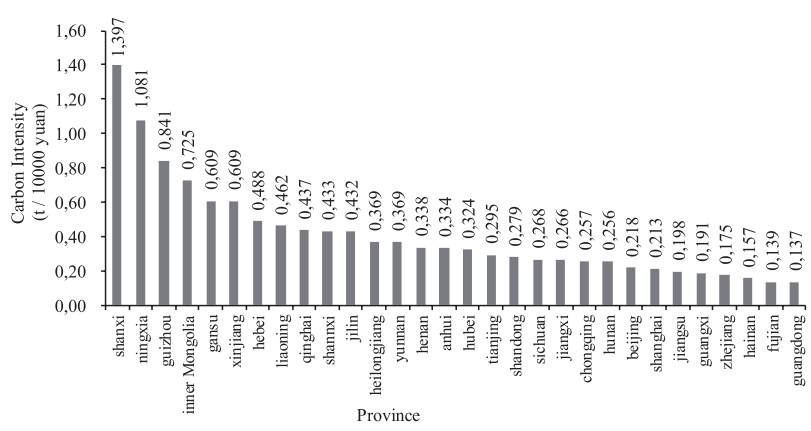

Fig. 3. Average CI of each province in China during 1995-2014. (in 1995 figures).

a minimum range of $19.74 \%$ to $59.05 \%$, while TO has a maximum range of $0.34 \%$ to $205.12 \%$.

\section{Results and Discussion}

\section{Changes in CI of China}

The CI in China over the period 1995-2014 has been calculated as shown in Fig. 1. The horizontal axis represents the year while the vertical axis represents the CI. The values of CI in China decreased from 1995 to 2014. Specifically, China's CI has declined from 0.501 10,000 ton (tc) per 10,000 yuan in 1995 to 0.204 tc per 10,000 yuan in 2014, with an annual growth rate of $-4.62 \%$. In terms of an evolutionary trend in CI variation, the whole 20 years can be divided into three phases: 1995-2002, 2002-08, and 2008-14. Changes in CI were markedly different in each of these three phases. During the first period, China's CI dropped at an annual rate of $6.79 \%$; in the second period, China's CI increased from 2002 to 2005 and then decreased at an annual rate of $3.09 \%$; during the third period, China's CI decreased steadily at an annual rate of $6.91 \%$.

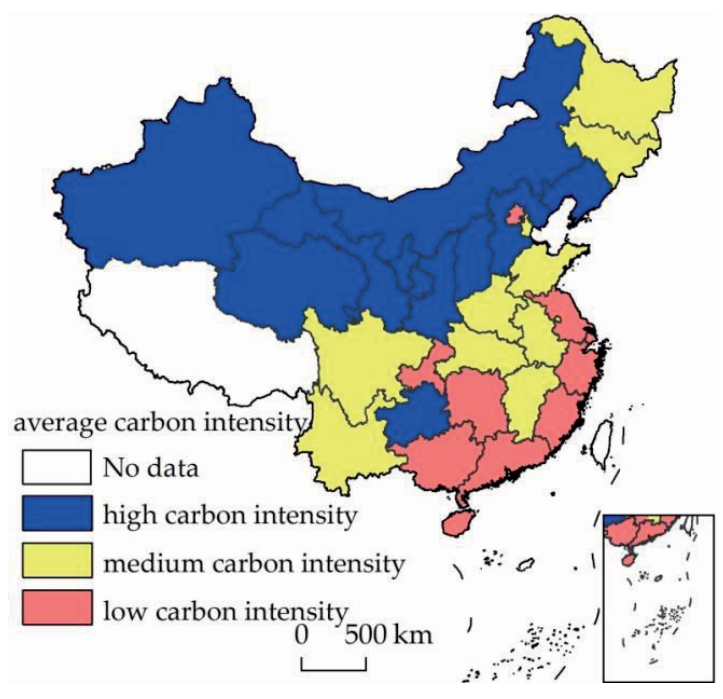

Fig. 4. Three regions with different grades of CI.

\section{Distribution of Carbon Intensity among Provinces in China}

The spatiotemporal CI dynamics in China for 19952014 are mapped in Fig. 2. CI was classified into four groups using the Jenks Natural Breaks Classification method [37], which is done by seeking to minimize the average deviation from the class mean while maximizing the deviation from the means of the other groups. Great disparities in CI have been identified in China, as shown in Fig. 2. In 1995 the CI in Shanxi was largest, exceeding 2.440 tc per 10,000 yuan, which is approximately 23.05 times the CI in Hainan, whose CI was lowest at approximately 0.106 tc per 10,000 yuan. With regard to the main groups of $\mathrm{CI}$ in this year, there are eight provinces in the first group, 15 in the second group, and six in the third group, with Cis of 0.683-1.086, 0.333-0.682, and $<0.332$, respectively.

In 2014 Ningxia, Shanxi, and Xinjiang ranked as the top three, and the values of $\mathrm{CI}$ in these three provinces were $1.322,0.856$, and 0.707 tc per 10,000 yuan, respectively. Moreover, the CI of Ningxia was 16.11 times larger than that of Beijing. At this time, the CI in 21 provinces, mainly located in Southeast China, was not more than 0.332 tc per 10,000 yuan. In contrast, provinces in northwestern China generally had higher CI values. In general, the provinces with low CI were usually located in southern China, while those with high CI were mainly located in northwestern China.

Furthermore, the average CIs of 30 provinces during 1995-2014 are illustrated in descending order in Fig. 3. Average CI varied greatly among different provinces in China. Specifically, Shanxi, Ningxia, and Guizhou ranked as the top three for average CI. Conversely, Hainan, Fujian, and Guangdong had the lowest average CI. In particular, Shanxi's average CI was 10.17 times larger than Guangdong's. Considering the research objective of this paper, we improved the classification method that was adopted by Chen [4]. To be specific, we used CI instead of energy consumption per capita as the indicator and divided 30 provinces into three regions: low, medium-, and high-level. The results of this grouping are (Fig. 4):

- Low-level CI provinces: Chongqing, Hunan, Beijing, Shanghai, Jiangsu, Guangxi, Zhejiang, Hainan, Fujian, and Guangdong.

- Medium-level CI provinces: Jilin, Heilongjiang, Yunnan, Henan, Anhui, Hubei, Tianjin, Shandong, Sichuan, and Jiangxi.

- High-level CI provinces: Shanxi, Ningxia, Guizhou, Inner Mongolia, Gansu, Xinjiang, Hebei, Liaoning, Qinghai, and Shannxi

Fig. 4 shows that provinces with low average CI were usually located in southeastern China, whereas the provinces with high average $\mathrm{CI}$ were mainly distributed in northern China. These three regions will be used to identify the influencing factors of CI separately on the basis of panel data. 
Table 2. Cross-province differences in carbon intensities through summary indices from 1995 to 2014.

\begin{tabular}{|c|c|c|c|c|c|}
\hline Year & CV & Gini & Year & CV & Gini \\
\hline 1995 & 0.693 & 0.314 & 2005 & 0.719 & 0.345 \\
\hline 1996 & 0.696 & 0.321 & 2006 & 0.724 & 0.348 \\
\hline 1997 & 0.688 & 0.331 & 2007 & 0.710 & 0.342 \\
\hline 1998 & 0.704 & 0.329 & 2008 & 0.701 & 0.342 \\
\hline 1999 & 0.658 & 0.320 & 2009 & 0.721 & 0.350 \\
\hline 2000 & 0.668 & 0.322 & 2010 & 0.735 & 0.351 \\
\hline 2001 & 0.687 & 0.324 & 2011 & 0.817 & 0.373 \\
\hline 2002 & 0.734 & 0.330 & 2012 & 0.831 & 0.383 \\
\hline 2003 & 0.808 & 0.363 & 2013 & 0.874 & 0.400 \\
\hline 2004 & 0.728 & 0.343 & 2014 & 0.900 & 0.408 \\
\hline
\end{tabular}

\section{$\mathrm{CV}$ and Gini Coefficient of CI}

Regarding regional differences in CI within China, two benchmark indices of inequality have been calculated during the period 1995 to 2014 (Table 2). The results unanimously indicate a rise in inequalities from 1995 to 2014, suggesting that the overall gap in CI between various provinces in China has increased gradually. As shown in Table 2, CV increased steadily from 0.693 in 1995 to 0.900 in 2014. Similarly, the Gini coefficient rose steadily from 0.314 to 0.408 during the same period. Taking into account the trends of the CV and Gini coefficient, the whole period can be divided into two phases: 1995-2007 and 2008-14. In the first phase, the Gini coefficient fluctuated approximately 0.34. However, the Gini coefficient increased continuously from 0.342 in 2008 to 0.408 in 2014. Although the value of the CV was larger than that of the Gini coefficient over the period from 1995 to 2014, the variation trend of $\mathrm{CV}$ is similar to that of the Gini coefficient.

However, the particularity of the sub-period 200003 in the first phase should be noted. Table 2 shows that both the $\mathrm{CV}$ and the Gini coefficient increased stably during 2000-04. The underlying reason may be the implementation of a Western development strategy in 2000. In the following four years, 2000-03, energy consumption experienced a sharp increase, leading to a rapid rise in CI, and the regional difference in CI widened. For instance, energy consumption in Ningxia increased from 13.55 million tons of standard coal in 2000 to 19.80 million tons in 2001, with a growth rate of up to $46 \%$. Additionally, 2008 is unique in the study period. Since the outbreak of the financial crisis that year, China has actively promoted industrial restructuring to steadily reduce CI. However, both the CV and the Gini coefficient increased rapidly from 2009 to 2014, which indicates that the interregional differences in CI continued to expand over the same period. Moreover, the continued expansion of regional differences in $\mathrm{CI}$ poses new challenges for China's CI targets.

\section{Panel Unit Root Test Results}

To overcome the errors inherent in some test methods, we conducted panel unit root tests for variables using the LLC and IPS tests (the LLC test for common root, and the IPS test for individual root). If both the LLC test and the IPS test reject the null hypothesis of non-stationarity, all the variables were stationary; otherwise, the variables were non-stationary. The results of the unit root tests are shown in Table 3, showing that all variables had a unit root at the $5 \%$ level of significance and so were not stationary. However, the first-order difference sequences of all the variables were stationary, thereby rejecting the null hypothesis of non-stationarity at less than a $1 \%$ level of significance. Based on these findings, the panel data were tested to see whether there was a co-integrational relationship between variables.

\section{Panel Data Co-Integration Results}

When time span $\mathrm{T}$ is small, the Kao test outperforms the Pedroni test; when $\mathrm{T}$ is large ( $\mathrm{T}>20)$, the Kao test is worse than the Pedroni test [28]. In this study $\mathrm{T}=20$; therefore, both the Pedroni and Kao tests are used to test whether there is a long-term stable relationship among variables. The test results are presented in Table 4. In a small sample, short-term, econometric analysis, the results of the panel ADF statistic and the group ADF statistic are the best. When the test conclusion differs, the results of panel ADF statistic and group ADF statistic should be considered first [3]. As shown in Table 4, panel

Table 3. Results of unit root tests.

\begin{tabular}{|c|c|c|c|c|}
\hline & \multicolumn{2}{|c|}{ Llc (common root) } & \multicolumn{2}{c|}{ IPS (individual root) } \\
\hline $\operatorname{lnCEI}$ & -1.540 & 0.062 & 1.285 & 0.901 \\
\hline $\operatorname{lnEI}$ & -1.550 & 0.061 & 5.731 & 1.000 \\
\hline $\operatorname{lnCP}$ & 1.952 & 0.975 & 0.985 & 0.838 \\
\hline $\operatorname{lnSI}$ & -1.451 & 0.073 & 1.670 & 0.953 \\
\hline $\ln \mathrm{R} L$ & 1.158 & 0.877 & 8.196 & 1.000 \\
\hline $\operatorname{lnFDI}$ & -3.950 & 0.000 & -1.437 & 0.075 \\
\hline $\ln \mathrm{TO}$ & -0.886 & 0.188 & -0.615 & 0.269 \\
\hline $\mathrm{D}(\operatorname{lnCEI})$ & -12.151 & 0.000 & -11.990 & 0.000 \\
\hline $\mathrm{D}(\operatorname{lnEI})$ & -2.840 & 0.002 & -3.153 & 0.001 \\
\hline $\mathrm{D}(\operatorname{lnCP})$ & -14.758 & 0.000 & -14.020 & 0.000 \\
\hline $\mathrm{D}(\operatorname{lnSI})$ & -11.076 & 0.000 & -9.357 & 0.000 \\
\hline $\mathrm{D}(\ln \mathrm{ln} L)$ & -9.901 & 0.000 & -8.823 & 0.000 \\
\hline $\mathrm{D}(\operatorname{lnFDI})$ & -15.587 & 0.000 & -14.904 & 0.000 \\
\hline $\mathrm{D}(\operatorname{lnTO})$ & -16.825 & 0.000 & -13.673 & 0.000 \\
\hline
\end{tabular}


Table 4. Results of co-integration tests.

\begin{tabular}{|c|c|c|c|c|c|}
\hline & & & & \multirow{2}{*}{$\begin{array}{c}\text { Weighted } \\
\text { Statistic }\end{array}$} \\
\hline \multirow{5}{*}{ Pedroni test } & & Statistic & $\mathrm{p}$ & $\mathrm{p}$ \\
\hline & Panel v-Statistic & -0.957 & 0.831 & -3.362 & 1.000 \\
\cline { 2 - 6 } & Panel rho-Statistic & 4.636 & 1.000 & 4.385 & 1.000 \\
\cline { 2 - 6 } & Panel PP-Statistic & -4.534 & 0.000 & -8.671 & 0.000 \\
\cline { 2 - 6 } & Panel ADF-Statistic & -2.580 & 0.005 & -3.917 & 0.000 \\
\cline { 2 - 6 } & Group rho-Statistic & 6.199 & 1.000 & & \\
\cline { 2 - 6 } & Group PP-Statistic & -10.960 & 0.000 & & \\
\hline
\end{tabular}

ADF, group ADF, panel PP, and group PP tests all reject the null hypothesis that there is no co-integration relationship at the 5\% significance level. Thus, it is supported that there is a co-integration relationship among CI and other independent variables. The results of the Kao test shown in Table 4 also reject the null hypothesis, which further supports the conclusion that there is a co-integrational relationship between variables.

\section{Parameter Estimation of the Panel Models}

From the measured results of the $\mathrm{CV}$ and the Gini coefficient mentioned above, the interregional differences in CI become wider. To further study the factors influencing CI, this paper has applied panel data models. First, all the data from the 30 provinces were used to build model 1 simulating the whole of China. Then, models 2 through 4, representing the regions of low-level, mediumlevel, and high-level CI, were set up with the data from each region.
Panel models often violate standard ordinary least squares (OLS) assumptions [38]. Three post-estimation tests for autocorrelation, cross-sectional independence, and group-wise heteroscedasticity for the 30 panels were performed. The result of the Wooldridge test [39] for autocorrelation in the panel data showed evidence of autocorrelation in the idiosyncratic errors, as it was $F(1,29)=144.979, p=0$. The $p$-value rejects the hypothesis $\mathrm{H}_{0}$ of no autocorrelation. The Pesaran test [40] for crosssectional independence in a fixed effects regression model clearly indicated that cross-sectional independence is proven (Pesaran's test of cross-sectional independence $=11.365, \mathrm{p}=0$ ). The modified Wald test [41] for groupwise heteroskedasticity in a fixed effect regression model clearly indicated that disturbances are heteroscedastic $(\chi 2(30)=6,720.51, p=0$, which rejects the hypothesis $\mathrm{H}_{0}$ of homoskedasticity). The above tests indicate that the optimal method choice is feasible generalized least squares (FGLS). The regression results from all four models are listed in Table 5.

Table 5. Results of regression of models.

\begin{tabular}{|c|c|c|c|c|c|c|c|c|}
\hline \multirow{4}{*}{ Independent variable } & \multicolumn{8}{|c|}{ Dependent variable (LNCEI) } \\
\hline & China & & Low & & Medium & & High & \\
\hline & model1 & & model2 & & model3 & & model4 & \\
\hline & Coef. & $\mathrm{p}$ & Coef. & $\mathrm{p}$ & Coef. & $\mathrm{p}$ & Coef. & $\mathrm{p}$ \\
\hline $\ln E L$ & -0.764 & 0.000 & -0.610 & 0.000 & -0.678 & 0.000 & -0.551 & 0.000 \\
\hline $\operatorname{lnCP}$ & 0.459 & 0.000 & 0.171 & 0.062 & 0.856 & 0.000 & 1.086 & 0.000 \\
\hline $\operatorname{lnSI}$ & 0.672 & 0.000 & 0.378 & 0.000 & 0.513 & 0.000 & 0.923 & 0.000 \\
\hline $\ln U R L$ & 1.503 & 0.000 & 1.479 & 0.000 & 1.009 & 0.000 & 0.888 & 0.000 \\
\hline $\operatorname{lnFDI}$ & -0.242 & 0.000 & 0.016 & 0.652 & -0.129 & 0.000 & -0.128 & 0.000 \\
\hline $\ln \mathrm{TO}$ & -0.126 & 0.000 & -0.193 & 0.000 & 0.139 & 0.000 & 0.004 & 0.841 \\
\hline $\mathrm{C}$ & 1.218 & 0.000 & -1.266 & 0.011 & 2.613 & 0.000 & 2.621 & 0.000 \\
\hline Observation & 600 & & 200 & & 200 & & 200 & \\
\hline
\end{tabular}




\section{Determinants of CI from a National Perspective}

In model 1 (see Table 5), the estimated coefficients of all variables were statistically significant, and the results conform to those from previous research. The estimated coefficients of EL, FDI, and TO were negative, which indicated that increasing economic development, foreign direct investment and trade openness will decrease China's CI. Among these three variables, economic development is the most important factor, with elasticity of -0.764 , indicating that a $1 \%$ increase in economic level would lead to a $0.764 \%$ decrease in CI when other factors remain constant. In contrast, the regression coefficients of CP, SI, and URL were positive, which reflected the fact that a rise in industrial proportion, coal consumption, and urbanization level will increase CI. Furthermore, the regression coefficient of URL is 1.503 , the largest of the coefficients, which means that rapid urbanization will result in a marked increase in CI. Over the past decades, China has experienced rapid urbanization. Specifically, the urbanization level has increased rapidly from $26.4 \%$ in 1990 to $54.7 \%$ in 2014 , and is expected to reach $60 \%$ by 2020 [42]. It is recognized that urbanization will lead to an increase in direct and indirect household carbon emissions [43], and consequently result in higher CI. In addition, the quality of urbanization in China is relatively low, and the process of urbanization is accompanied by a large amount of energy consumption, which also has contributed to an increase in CI.

Generally, it was found that economic development, foreign direct investment, and trade openness can decrease CI. Additionally, the decline in the industrial proportion and coal consumption will also inhabit CI. However, the rise in urbanization inevitably increases CI. Accordingly, to achieve its CI reduction targets, China should sustainably develop its economy, vigorously adjust its industrial structure, optimize its energy structure, and continue to improve trade openness. Because the urbanization level proved to be the most important factor affecting China's CI, China should pay attention to the quality rather than the speed of urbanization.

\section{Determinants of CI from a Regional Perspective}

In the low-level region model 2 , the estimated coefficients of EL, SI, URL, and TO were statistically significant. The regression coefficient of URL is the largest, followed by economic development (absolute value). Therefore, controlling the speed of urbanization rationally and developing its economy sustainably are essential for $\mathrm{CI}$ reduction in this region. Moreover, in the medium-level region model 3 , the estimated coefficients of all variables were also statistically significant. In this model, the regression coefficient of URL is the largest, with a value of 1.009 , which indicates that urbanization level is the most important factor promoting the increase in CI. Moreover, energy structure (CP) was followed by
URL. Accordingly, improving the quality of urbanization and optimizing the energy structure is the medium-level region's top priority to achieve low-carbon development. Finally, in the high-level region model 4, the estimated coefficients of variables were all statistically significant, with the exception of TO. Different from model 2 and model 3, the regression coefficient of $\mathrm{CP}$ is the greatest in model 4, followed by industrial structure (SI). Hence adjusting the industrial structure and optimizing the energy structure are urgent tasks for the high-level region to achieve CI reduction targets. In summary, the dominant factors that affect CI vary widely across regions. Furthermore, to reveal the intrinsic mechanism of the variation of the coefficient of the same variable in different regions, it is necessary to conduct a concrete and in-depth analysis of each specific variable. The regression coefficients of EL in all models were negative and statistically significant, which provided sufficient evidence of the role of economic development in promoting a lower CI. Specifically, the regression coefficients of EL were -0.610 in the low-level region, -0.678 in the medium-level region, and -0.551 in the highlevel region. From the absolute value of EL's coefficient, EL is lowest in the high-level region and highest in the medium-level region, which illustrates that the potential for economic development on CI reduction is greatest in the medium-level region.

As for the variable $\mathrm{CP}$, the regression coefficients of this variable in all models were positive, but not statistically significant at the $5 \%$ significance level in model 2. As Table 5 shows, energy structure has a strong impact on CI in both the medium- and high-level regions. However, the effect of energy structure on CI in the lowlevel region was not significant. Moreover, the regression coefficient of $\mathrm{CP}$ in the high-level region was greater than that in the medium-level region, which indicated that the potential for energy structure adjustment on CI reduction is greatest in the high-level region. Take 2014, for instance: the proportion of coal consumption was $61.50 \%$ in the medium-level region, and it was up to $69.24 \%$ in the high-level region.

With regard to the variables SI and URL, the regression coefficients of these two variables in all models were positive and statistically significant. To be specific, the coefficient of SI in model 4 is the greatest, as is the coefficient of URL in model 2. The results show that the impact of industrial structure on CI is greatest in the high-level region when compared to the other two regions. Take 2014 as an example: the industrial proportion was $44.26 \%$ in the low-level region, $48.56 \%$ in the medium-level region, and $49.52 \%$ in the high-level region. In other words, this implies that higher industrial proportion is always accompanied by higher energy consumption, thus leading to higher CI. In addition, the urbanization level played the most important role in CI growth in the low-level region.

As for the last two factors, TO and FDI, these two variables were used together to stand for trade liberalization. It can be seen from Table 5 that the 
effects of FDI and TO on CI reduction were different in different models. The estimated coefficient of foreign direct investment (FDI) was not significant in model 2, which indicated that there was no significant relationship between FDI and CI in the low-level region. Although many scholars believe that FDI can effectively promote the reduction of CI in China [44], the results from our paper show that the effect of FDI on reducing CI was very limited in the low-level region. The underlying cause may be the variation in technology spillovers, which weaken gradually along with economic growth. However, FDI has a positive effect on CI reduction in both the medium-level and high-level regions, which means that an increase in FDI will inhibit CI growth. With regard to the variable $\mathrm{TO}$, the estimated coefficient of TO was negative and statistically significant in model 2, whereas it was positive and statistically significant in model 3. However, it was not statistically significant in model 4. In other words, the impact of trade openness on $\mathrm{CI}$ is indefinite, and the main reason may lie in the significant regional differences in types of import and export products. Specifically, the main import and export products in the low-level region are always high valueadded products. In contrast, raw materials and low valueadded products were the main goods for import and export trade in the medium- and low-level regions. Thus, the higher the total import and export volume, the higher the CI.

\section{Conclusions}

\section{Major Conclusions}

In this paper we first calculate the values of CI in 30 provinces in China during the period from 1995 to 2014. Then, by combining the CV and the Gini coefficient, this paper further analyzed the spatiotemporal CI dynamics in those 30 provinces and classified all provinces into three regions: low-, medium-, and high-level. Finally, by applying the panel data and FGLS method, we identified the influencing factors of $\mathrm{CI}$ at both the national and regional levels. The results are:

1) The values of CI in China decreased from 0.501 tc per 10,000 yuan in 1995 to 0.204 tc per 10,000 yuan in 2014 , with an annual growth rate of $-4.62 \%$.

2) During the study period, the $\mathrm{CV}$ increased steadily from 0.693 to 0.900 , and the Gini coefficient also increased steadily from 0.314 to 0.408 . This indicated that the difference between provinces in CI has increased gradually, posing a challenge for achieving China's CI reduction target.

3) From the national perspective, it was found that economic level, foreign direct investment and trade openness had a negative effect on CI, indicating that these three factors acted as disincentives to CI. In contrast, both coal consumption and industrial proportion played a role in increasing CI. In addition, the urbanization level was proven to be the most important factor in $\mathrm{CI}$, which indicated that $\mathrm{CI}$ in China can be reduced by controlling the pace of urbanization.

4) From the regional perspective, the dominant factor in CI varied by region. Specifically, in the low-level region, the dominant factor influencing CI is urbanization, followed by economic level; in the medium-level region, the two most important influencing factors are urbanization level and coal consumption; in the highlevel region, the top two factors are coal consumption and industrial proportion. In summary, there are significant differences in the development of different regions, which largely explained the differences in the relationships between the variables. Thus, each region should pay more heed to its own particular characteristics.

\section{Policy Implications}

China has promised that its carbon dioxide emissions will peak in 2030, and CI will then be reduced by $60-65 \%$ compared to the level in 2005 . According to the conclusions drawn in this study, policy suggestions are proposed as follows:

1) The difference in CI between provinces in China has consistently grown wider. Thus, using the same carbon reduction goal for all the provinces is not only unscientific but also unfair. In consideration of the tremendous differences, when setting CI reduction targets, policymakers must take into account the specific situation of each province. The provinces with low average CI were usually located in southeastern China, while the provinces with high average CI were mainly located in northern China. Considering the need for winter heating in northern China, the CI target in this region should be slightly higher.

2) From the national perspective, urbanization level is the most important factor affecting China's CI. It is the quality of urbanization that matters, not the pace. In China, the government has a major impact on urbanization development and urban planning. Therefore, moving forward with the pilot project to build low-carbon cities and using effective space-time allocation of land resources will effectively guide the development pattern. In particular, the government should: 1) establish an incentive mechanism for landintensive use and prevent new urban construction from being over-dependent on resource consumption, which may lead to low-quality urbanization; 2) carefully manage the relationship between urban space expansion and ecological environmental protection and reasonably demarcate the border of urban growth; and 3) reduce carbon emissions, use measures such as reducing surface hardening, making central heating available in communities, constructing green buildings and low-carbon infrastructure, reducing traffic congestion, and increase the proportion of urban public green space. 
3) From the national perspective, different measures need to be taken in different CI regions. In low-level regions, improvement in the quality of urbanization and economic development are most important. Hence, in this region possible solutions to CI reduction include giving full play to the role of human capital, developing high-tech small and medium enterprises (SMEs), and giving more emphasis to commercial and financial services. Vigorously optimizing the energy structure is the most urgent task in the medium-level region. Improving energy efficiency is also needed in this region. Finally, in the high-level region, the most effective way to reduce $\mathrm{CI}$ is optimizing the industrial structure. Specifically, the government should renovate and upgrade traditional industries; reduce synthetic energy consumption by industry; limit the development of iron, steel, cement, and other highenergy-consuming industries; and raise industry access standards. It should also guide and cultivate tertiary industry, raising the proportion of tertiary industry in regional economies.

\section{Acknowledgements}

Our current work is supported by the Grant Program of Clean Development Mechanism Fund of China (No. 2014092) and the Chinese Postdoctoral Science Foundation (2016M600121).

\section{References}

1. SAUTER C., GRETHER J.M., MATHYS N.A. Geographical spread of global emissions: Within-country inequalities are large and increasing. Energy Policy, 89, 138, 2016.

2. World energy outlook 2016. International Energy Agency, 2016, Available online: http://www.iea.org (accessed on 15 March 2017).

3. DONG F., LONG R., LI Z., DAI Y. Analysis of carbon emission intensity, urbanization and energy mix: evidence from China. Natural Hazards, 82, 1375, 2016.

4. CHEN J., WU Y., WEN J., CHENG S., WANG J. Regional differences in China>s fossil energy consumption: an analysis for the period 1997-2013. Journal of Cleaner Production, 142, 578, 2017.

5. DU K., LIN B., XIE C. Exploring Change in China's Carbon Intensity: A Decomposition Approach. Sustainability, 9, 296, 2017.

6. WANG S., FANG C., WANG Y. Spatiotemporal variations of energy-related $\mathrm{CO}_{2}$ emissions in China and its influencing factors: An empirical analysis based on provincial panel data. Renewable and Sustainable Energy Reviews, 55, 505, 2016.

7. GUO F., ZHAO T., WANG Y., WANG Y. Estimating the abatement potential of provincial carbon intensity based on the environmental learning curve model in China. Natural Hazards, 84, 685, 2016.

8. ZHU Z.S., LIAO H., CAO H.S., WANG L., WEI Y.M., YAN $\mathrm{J}$. The differences of carbon intensity reduction rate across
89 countries in recent three decades. Applied Energy, 113, 808, 2014

9. WANG H., YANG Y., KELLER A.A., LI X., FENG S.J., DONG Y.N., LI F.T. Comparative analysis of energy intensity and carbon emissions in wastewater treatment in USA, Germany, China and South Africa. Applied Energy, 184 (12), 873, 2016.

10. DURO J.A. The international distribution of energy intensities: Some synthetic results. Energy Policy, 83, 257, 2015.

11. CHENG Y., WANG Z., YE X., WEI Y.D. Spatiotemporal dynamics of carbon intensity from energy consumption in China. Journal of Geographical Sciences, 24, 631, 2014.

12. DURO J.A. On the automatic application of inequality indexes in the analysis of the international distribution of environmental indicators. Ecological Economics, 76, 1, 2012.

13. DURO J.A., ALCÁNTARA V., PADILLA E. International inequality in energy intensity levels and the role of production composition and energy efficiency: An analysis of OECD countries. Ecological Economics, 69, 2468, 2010.

14. DURO J.A. Intercountry inequality on greenhouse gas emissions and world levels: An integrated analysis through general distributive sustainability indexes. Ecological Indicators, 66, 173, 2016.

15. YUE C., HU X., CANFEI H., ZHU J., WANG, S., FANG J. Provincial Carbon Emissions and Carbon Intensity in China from 1995 to 2007 (Carbon Emissions and Social Development III). Acta Scientiarum Naturalium Universitatis Pekinensis, 46, 510, 2010 [In Chinese].

16. LI J., HUANG X., YANG H., CHUAI X., WU C. Convergence of carbon intensity in the Yangtze River Delta, China. Habitat International, 60, 58, 2017.

17. ZHAO X., WESLEY B.J., LACOMBE D.J. Province-level convergence of China's carbon dioxide emissions. Applied Energy, 150, 286, 2015.

18. ZHANG D., BROADSTOCK D.C. Club Convergence in the Energy Intensity of China. Energy Journal, 37 (3), 137, 2016.

19. BURNETT J.W., MADARIAGA J. The convergence of U.S. state-level energy intensity, Energy Economics, 62 (2), 357, 2017.

20. HERRERIAS M.J., ALLER C., ORDÓÑEZ J. Residential energy consumption: A convergence analysis across Chinese regions. Energy Economics, 62 (2), 371, 2017.

21. HERRERIAS M.J. World energy intensity convergence revisited: A weighted distribution dynamics approach. Energy Policy, 49, 383, 2012.

22. ZHANG W., LI K., ZHOU D., ZHANG W., GAO H. Decomposition of intensity of energy-related $\mathrm{CO}_{2}$ emission in Chinese provinces using the LMDI method. Energy Policy, 92, 369, 2016.

23. WANG J., ZHAO T., XU X., ZHANG X. Exploring the changes of energy-related carbon intensity in China: an extended Divisia index decomposition. Natural Hazards, 83, 501, 2016.

24. XIONG C.H., YANG D.G., HUO J.W., ZHAO Y.N. The relationship between agricultural carbon emissions and agricultural economic growth and policy recommendations of a low-carbon agriculture economy in Hotan Prefecture, China. Polish Journal of Environmental Studies. 25 (5), 2187, 2016.

25. SUPASA T, HSIAU S.S., LIN S.M., WU. J.C. Has energy conservation been an effective policy for Thailand? An input-output structural decomposition analysis from 1995 to 2010. Energy Policy, 98, 210, 2016. 
26. SU B., ANG B.W. Multiplicative decomposition of aggregate carbon intensity change using input-output analysis. Applied Energy, 154, 13, 2015.

27. XIE Y., WENG Q. World energy consumption pattern as revealed by DMSP-OLS nighttime light imagery, Giscience \& Remote Sensing, 53 (2), 265, 2015.

28. WANG Z., ZHANG B., LIU T. Empirical analysis on the factors influencing national and regional carbon intensity in China. Renewable and Sustainable Energy Reviews, 55, 34, 2016.

29. ROBAINA A.M., MOUTINHO V. Decomposition analysis and Innovative Accounting Approach for energy-related $\mathrm{CO}_{2}$ (carbon dioxide) emissions intensity over 1996-2009 in Portugal. Energy, 57, 775, 2013.

30. CHEN J., CHENG S., SONG M., WANG J. Interregional differences of coal carbon dioxide emissions in China. Energy Policy, 96, 1, 2016.

31. WANG Z., YANG L. Delinking indicators on regional industry development and carbon emissions: BeijingTianjin-Hebei economic band case. Ecological Indicators, 48, 41, 2015.

32. OU J., LIU X., LI X., CHEN Y. Quantifying the relationship between urban forms and carbon emissions using panel data analysis. Landscape ecology, 28, 1889, 2013.

33. YORK R., ROSA E.A., DIETZ T. Footprints on the earth: The environmental consequences of modernity. American sociological review, 68 (2), 279, 2003.

34. ALPER A., ONUR G. Environmental Kuznets curve hypothesis for sub-elements of the carbon emissions in China. Natural Hazards, 82 (2), 1327, 2016.

35. The National Bureau And Statistics of China. National Statistics Yearbook of China. China Statistics Press: Beijing, China, 1996-2015.

36. Ministry of Commerce of the Peopless Republic of China. China Statistical Yearbook of Commerce. China Statistics Press: Beijing, China, 1996-2015.
37. KALANTARI M., YAGHMAEI B., GHEZELBASH S. Spatio-temporal analysis of crime by developing a method to detect critical distances for the Knox test, International Journal of Geographical Information Science, 30 (11), 2302, 2016.

38. LAKKA S., STAMATI T., MICHALAKELIS C., MARTAKOS D. What drives eGovernment growth? An econometric analysis on the impacting factors. International Journal of Electronic Governance, 6, 20, 2013.

39. WOOLDRIDGE J.M. Econometric analysis of cross section and panel data, The MIT press: London, England, 2010.

40. PESARAN M.H. General Diagnostic Tests for Cross Section Dependence in Panels. Cambridge Working Papers in Economics, 7, 1240, 2004.

41. MARTORANO B., SANFILIPPO M. Structural Change and Wage Inequality in the Manufacturing Sector: Long Run Evidence from East Asia. Oxford Development Studies, 43 (2), 212, 2015.

42. The State Council of the People's Republic of China. National guidelines for developing a new type of urbanization, 2014. Available online: http://money.163. com/14/0317/08/9NHA7U7300253B0H.html (accessed on 22 March 2017)

43. LI Y., ZHAO R., LIU T., ZHAO J. Does urbanization lead to more direct and indirect household carbon dioxide emissions? Evidence from China during 1996-2012. Journal of Cleaner Production, 102, 103, 2015.

44. ZHANG C., ZHOU X. Does foreign direct investment lead to lower $\mathrm{CO}_{2}$ emissions? Evidence from a regional analysis in China, Renewable \& Sustainable Energy Reviews, 58, 943, 2016. 